\title{
Bridging the Gap: Improving Clinical Development and the Regulatory Pathways for Health Products for Neglected Diseases
}

\section{Thomas J. Bollyky}

\section{Abstract}

There has been tremendous progress over the last decade in the development of health products for neglected diseases. These include drugs, vaccines, and diagnostics for malaria and tuberculosis, which kill millions of people annually, plus other diseases like chagas and dengue fever, which may less familiar, but nonetheless exact a large and often lethal toll in the world's poorest communities. Led by product development publicprivate partnerships (PDPs) and fueled by the support of the Bill \& Melinda Gates Foundation, the National Institutes of Health, and other donors, there are now dozens of candidate products in the pipeline.

Two substantial bottlenecks, however, threaten our capacity to bring these products to those in need. First, the research and regulatory capacity in many neglected disease-endemic settings is not adequate to support the clinical trials that need to occur there in order to complete the development of these products. Second, even with expected attrition in the pipeline, current levels of financing are insufficient to support the clinical development of these products under current cost assumptions. Addressing these related challenges requires not only increased funding for large scale clinical trials and capacity building, but also greater attention to how these trials and their regulatory pathways can be improved to reduce unnecessary costs, delays, and risks to trial subjects.
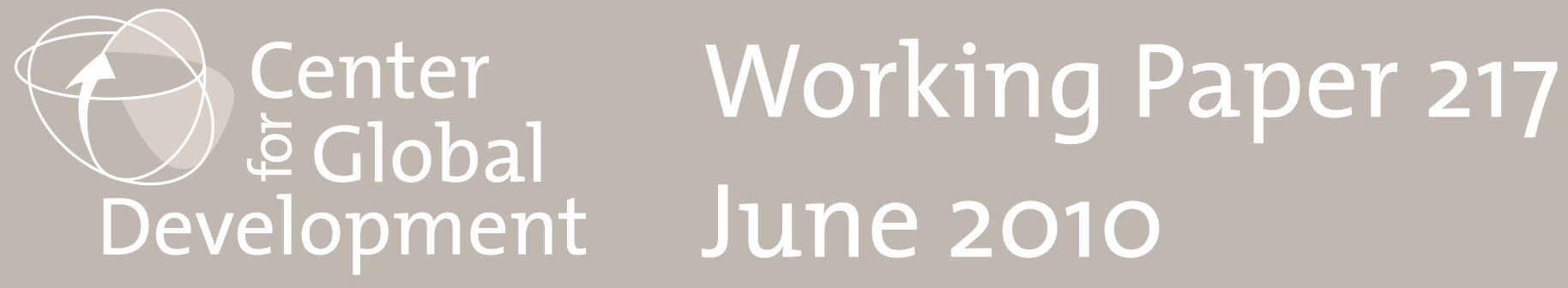


\title{
Bridging the Gap: Improving Clinical Development and the Regulatory Pathways for Health Products for Neglected Diseases
}

\author{
Thomas J. Bollyky \\ Center for Global Development
}

Thomas J. Bollyky is a visiting fellow at the Center for Global Development. He would like to thank Drs. Ernst Berndt, Fabio Thiers, and, especially, Iain Cockburn from the National Bureau of Economic Research for their tireless support in the preparation of this report. He would also like to express his appreciation for Ruth Levine and his Center for Global Development colleagues_-Vijaya Ramachandran, Steve Rosenzweig, and David Wheeler in particular-for their patient assistance.

This paper was drafted as part of a launch of a CGD Initiative on Better, Faster, and Cheaper Clinical Trials for Health Products for Neglected Diseases. CGD is grateful for contributions from the the Bill \& Melinda Gates Foundation in support of this work.

Thomas J. Bollyky. 2010. "Bridging the Gap: Improving Clinical Development and the Regulatory Pathways for Health Products for Neglected Diseases." CGD Working Paper 217. Washington, D.C.: Center for Global Development.

http://www.cgdev.org/content/publications/detail/1424250

Center for Global Development 1800 Massachusetts Ave., NW Washington, DC 20036

202.416.4000

(f) 202.416 .4050

www.cgdev.org
The Center for Global Development is an independent, nonprofit policy research organization dedicated to reducing global poverty and inequality and to making globalization work for the poor. Use and dissemination of this Working Paper is encouraged; however, reproduced copies may not be used for commercial purposes. Further usage is permitted under the terms of the Creative Commons License.

The views expressed in this paper are those of the author and should not be attributed to the board of directors or funders of the Center for Global Development. 


\section{Executive Summary}

There has been tremendous progress over the last decade in the development of health products for neglected diseases. These include drugs, vaccines, and diagnostics for malaria and tuberculosis, which kill millions of people annually, plus other diseases like chagas and dengue fever, which may less familiar, but nonetheless exact a large and often lethal toll in the world's poorest communities. ${ }^{1}$ Led by product development public-private partnerships (PDPs) and fueled by the support of the Bill \& Melinda Gates Foundation, the National Institutes of Health, and other donors, there are now dozens of candidate products in the pipeline. $^{2}$

Two substantial bottlenecks, however, threaten our capacity to bring these products to those in need. First, the research and regulatory capacity in many neglected disease-endemic settings is not adequate to support the clinical trials that need to occur there in order to complete the development of these products. Second, even with expected attrition in the pipeline, current levels of financing are insufficient to support the clinical development of these products under current cost assumptions. Addressing these related challenges requires not only increased funding for large scale clinical trials and capacity building, but also greater attention to how these trials and their regulatory pathways can be improved to reduce unnecessary costs, delays, and risks to trial subjects.

This paper proceeds in three parts.

The first section provides a brief overview of the clinical development process and the sources of its increasing cost, duration, and uncertainty. These include: the growth and complexity of clinical trial regulation; the widespread adoption of high-cost, arguably inefficient commercial clinical development practices; and the growing sophistication of the candidate biopharmaceuticals themselves.

The next section examines the particular challenges of clinical development of health products for neglected diseases. These include: the necessity of running trials in disease-

\footnotetext{
1 This paper defines neglected diseases broadly to refer malaria, TB, dengue fever, treponematoses, leptospirosis, strongyloidiasis, foodborne trematodiases, neurocysticercosis, scabies, thirteen parasitic and bacterial infections; three soil-transmitted helminth infections (ascariasis, hookworm, and trichuriasis), lymphatic filariasis, onchocerciasis, dracunculiasis, schistosomiasis, Chagas disease, human African trypanosomiasis, leishmaniasis, Buruli ulcer, leprosy, and trachoma.

2 See, e.g., Tufts Center for the Study of Drug Development, Drug Approvals for neglected diseases increase along with more R\&D Funding, 11 IMPACT REPORT (2009) (concluding there are 74 products to treat neglected disease currently in the clinical development pipeline).
} 
endemic settings with limited clinical research capacity and under-developed regulatory systems; the complexity of the diseases themselves; the challenges of working with large numbers of otherwise healthy, vulnerable, often pediatric subjects; and, finally, the need to conduct trials in multiple sites and jurisdictions with overlapping regulatory and ethics approval and monitoring processes.

In the third, and final, section, this paper proposes a two-pronged strategy that the Center for Global Development (CGD) is pursuing to help bring the costs, risks, and finances for clinical trials for health products for neglected diseases into a better, more sustainable balance:

- Sensible Guidelines for Neglected Disease Clinical Trials. Complex products and diseases, vulnerable subjects, resource-poor research settings, and cost-sensitive circumstances demand better, faster, and cheaper clinical trials. Several promising initiatives are assessing large, multi-center clinical trials to evaluate how they may be modified to reduce their cost without sacrificing scientific rigor, quality assurance, or the protection of trial subjects and patients. By bringing together respected experts from academic centers, national regulatory authorities and institutional review boards, industry, and PDPs to adapt that emerging research to the needs of the neglected disease product pipeline and the challenges of the regulatory pathways for these products, we will seek to produce guidelines that offer a sustainable means of reducing clinical trials costs, risks, and delays for products for neglected diseases.

- Integrated and Streamlined Regulatory Pathways for Clinical Development. A single, regional pathway with integrated regulatory and ethics reviews for clinical trials would increase the efficiency with which products for neglected diseases are brought to market and to reduce costs. Based on the foregoing analysis and existing precedents for regional regulatory pathways, we propose a joint review/centralized procedure model. If well designed and sufficiently supported, regional regulatory cooperation on clinical research would pool scarce regulatory resources, limit inconsistencies and overlap, reduce the cost, duration, and risk of trials, and help attract investment in regulatory capacity building.

Vision, strategic investments, and hard work built the current pipeline of products for neglected diseases. Realizing the promise of that pipeline and ensuring its future vitality will require improved clinical trial practices and an analogous commitment to building regulatory pathways more favorable to trial subjects and current and future innovation. 


\section{Introduction}

Clinical trials are foundational to public health and medical innovation. They provide the evidentiary basis for the diagnosis, treatment, and prevention of disease.

This role is particularly important in the case of neglected diseases. Much remains unknown about the biology of many of these diseases. The genetic characteristics of the populations and socioeconomic settings in which these diseases are endemic can differ in substantial ways from those encountered in the developed world. The treatments, diagnostics, and preventative interventions currently in the product pipeline will be, for many neglected diseases, the first new tools in a generation and, for others, they will be simply the first. Clinical trials will be the means by which these innovative interventions are developed and the basis for the regulatory approval that they require before these products can be licensed and distributed to patients.

There are, however, factors that significantly increase the risk, delays, and cost of these clinical trials. Some are problematic for clinical trials generally. Others are particular to product development clinical trials for neglected diseases in disease-endemic countries.

\section{Challenges in the Clinical Development of Drugs and Vaccines Generally}

The figure below represents a simplified version of the clinical trial process for an innovative pharmaceutical product in the United States. ${ }^{3}$
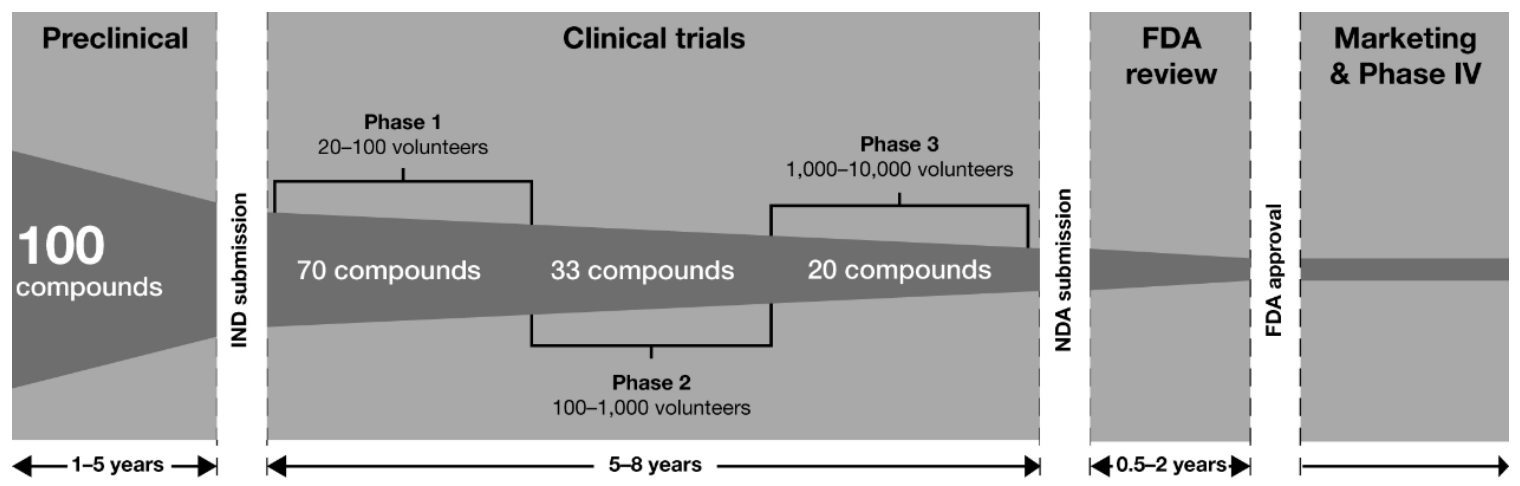

A prospective clinical trial sponsor must complete extensive safety/toxicity studies in animal models to establish that the investigational new drug will not expose human subjects to unreasonable risks when used in limited, early stage clinical studies. These studies may take one to five years. Once completed, a sponsor organization may file an Investigational New Drug (IND) application with the U.S. Food and Drug Administration (FDA). An IND may

\footnotetext{
3 The regulatory pathways and clinical development process for the various types of medical technology products - vaccines, drugs, devices, and diagnostics - are different. For instance, clinical development of medical devices and diagnostics are generally not as heavily regulated as drugs or vaccines. For the purposes of this general background, we focus on clinical development of drugs in the U.S.
} 
be filed for an unapproved product or for a new indication or patient population for an approved product. The IND must contain detailed information concerning the animal pharmacological and toxicology studies, the manufacturing of the product, the investigator, and the protocols for the proposed clinical trials. The IND must also include commitments from the sponsor to obtain informed consent from research subjects and a review of the study by an institutional review board (IRB), and to adhere to other U.S. IND regulations. Once the IND is submitted, the sponsor must wait 30 days before initiating any clinical trials. During this time, FDA has an opportunity to review and place a clinical hold on the IND. If no hold is placed, clinical testing may begin. ${ }^{4}$

Clinical trials are broadly categorized into four phases. ${ }^{5}$

Phase I trials are used to determine a dose with an acceptable level of safety and examine the biological and pharmacological effects of the product. These trials can last up to a year and usually involve a hundred subjects or fewer.

Phase II trials generate a preliminary estimate of a drug or vaccine's efficacy, safety, dose tolerability, and potential side effects. These trials typically involve several hundred to a few thousand subjects and last six months to two years. These trials are often run concurrently at multiple clinical trial sites in one or more countries.

Phase III trials are large scale trials intended to provide a more definitive answer on the safety and efficacy of the intervention. Subjects are usually randomly allocated (randomized) to intervention groups and the study drug or vaccine is assessed in comparison to a control (a known comparator product, often a placebo). These trials can involve hundreds or, more and more frequently, thousands of subjects and require three to five years to complete. It is often necessary to conduct more than one trial to test the product under varied conditions and different disease patterns, patient populations, or indications. If the phase III results demonstrate safety and sufficient efficacy, the manufacturer of the drug or vaccine can submit an application to the national regulatory authority to license and market the product in that country.

Phase IV trials are post-marketing surveillance studies or studies required as a condition of regulatory approval. These trials are used to monitor the safety and efficacy of the product, its duration of benefit, and to identify rare, serious adverse events that may not become evident until the drug or vaccine is used by many patients. These trials involve thousands of subjects in the general target population, rather than a selected group of subjects who agree to participate in the trial. These trials can last four to six years.

\footnotetext{
${ }^{4}$ An approved IND also allows a clinical trial sponsor to transport the investigational drug across state lines.

5 Over the years, these "phases" have started to break down and overlap. Sometimes, phase I and II trials are combined. Conversely, phase II trials are sometimes divided into Phase IIA trials, which assess dosing requirements, and Phase IIB, which study efficacy.

${ }^{6}$ In the U.S., the application is called a New Drug Application (NDA) for most synthesized molecules and a Biologic License Application (BLA) for most biologics.
} 
Clinical trials, for the development of drugs and vaccines in particular, have become increasingly time-consuming and expensive. The process typically lasts between 10-14 years. Clinical trial costs account for as much as 70 percent of the total cost of developing a product. Per subject costs can be as high as $\$ 30,000$. The figure below illustrates an estimate of the growth in total investment required to launch a successful drug over two different time periods. Most of the increased cost of the "critical path" period depicted results from clinical development costs.

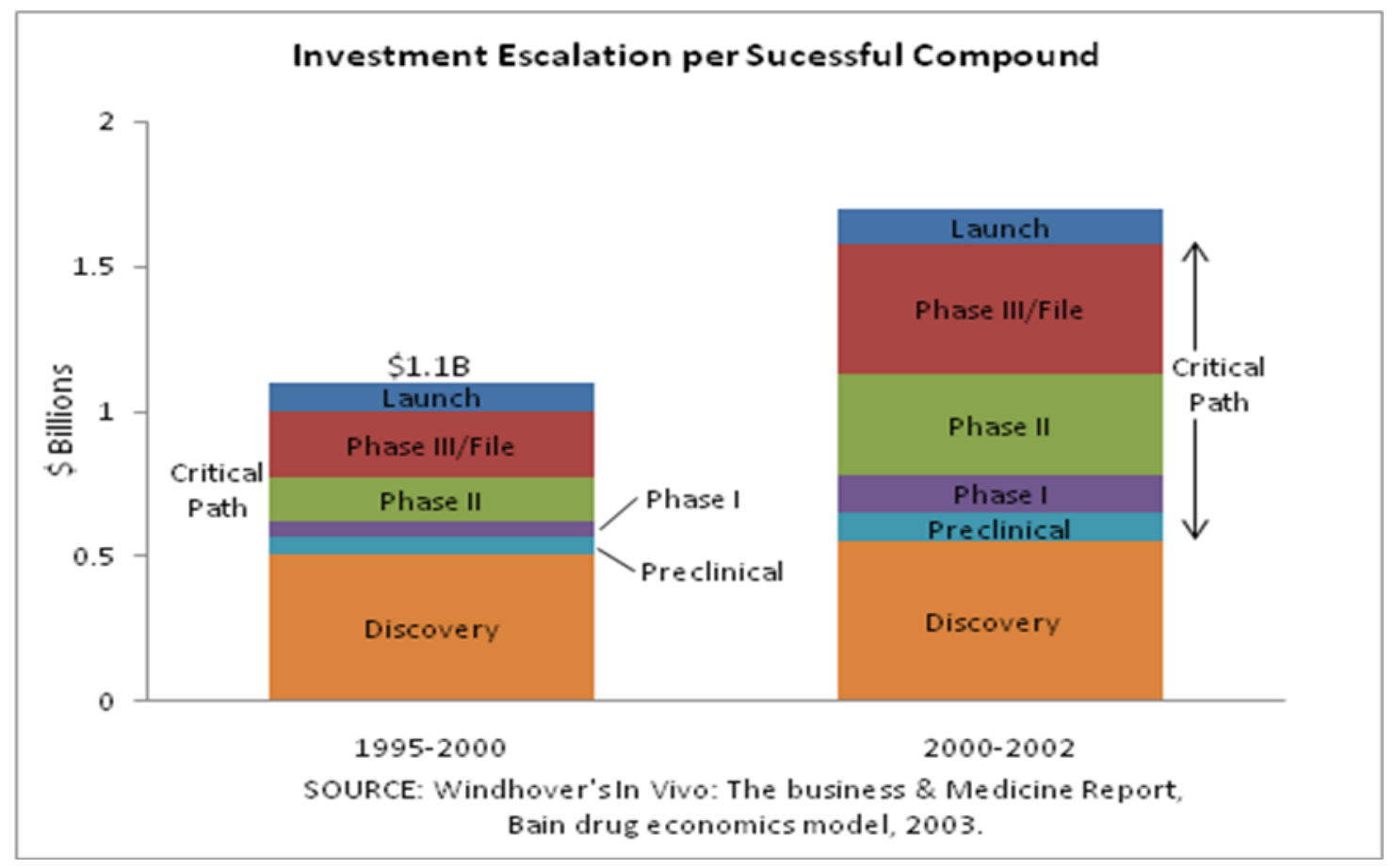

While costs have increased, the productivity of product development clinical trials has steadily eroded. ${ }^{7}$ Between 1991 and 2003, the costs of clinical development increased seven percent per year after adjusting for inflation, while there was a 34 percent reduction in the number of new drugs approved in same time period. ${ }^{8}$ Most biopharmaceutical research and development $(\mathrm{R} \& \mathrm{D})$ projects fail, with the candidate medicine never making it to market. For every 100 drugs for which an IND application is submitted to the FDA, 70 will successfully complete Phase I clinical testing, 33 will successfully complete phase II clinical testing and proceed to phase III, and, of those, only 20 will be approved for marketing.' The success rates for clinical development of vaccines are usually even lower.

\footnotetext{
7 See Government Accountability Office (GAO), New Drug Development: Science, Business,

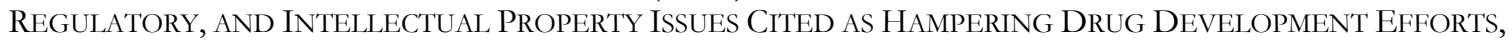
GAO-07-49 15 (2006) (indicating that the rate of NDAs fell over 38 percent between 1994-2004).

${ }^{8}$ Eric Eisenstein et al., Sensible approaches for reducing clinical trials, ClinICAL TRIALS 75, 83 (2008) but see Ernst Berndt et al., The Impact of Incremental Innovation in Biopharmaceuticals: Drug Utilisation in Original and Supplemental Indications, 2 PHARMACOECONOMICS 69 (2006) (arguing increases in approvals of new dosages, formulations, or indications should not be discounted since these incremental innovations may have substantial health benefits). 9 Peter Barton Hutt et al., Food and Drug Law: Cases and Materials, 624 (3rd ed. 2007).
} 
The reasons for the increased cost and decreased productivity of clinical development are at least fourfold.

First, clinical trials have become more complicated and costly, in part, because the products and outcomes they are designed to evaluate are more complex. A large and growing proportion of investigational treatments target biologically complex chronic illnesses that require longer periods to effectively measure end points. ${ }^{10}$ Further, protocols for new biologics have more stringent eligibility requirements and necessitate more elaborate monitoring methods, such as diagnostic assessment of biomarkers, to evaluate safety and efficacy. It is now usually necessary to conduct more than one phase III trial.

Second, changes in clinical trial regulation have contributed to the growth of clinical trial duration and cost. ${ }^{11}$ Regulation of clinical trials is essential for ensuring the safety, wellbeing, and rights of clinical trial subjects and the validity of clinical data. However, since 1962, when the FDA and other national regulators began regulating the clinical development process, those regulations have tended to accrue, with new regulations adopted in response to specific scandals. ${ }^{12}$ Over time, these regulations have accumulated, layering on top of one another, with relatively little subsequent streamlining to address scientific advances. ${ }^{13}$

An example of this dynamic is clinical trial data monitoring and record keeping. These tools are important for protecting the rights and well-being of subjects and preventing clinical trial fraud. However, national and international requirements for data monitoring and record keeping have increased in complexity in response to episodes of clinical trial data fraud. ${ }^{14}$ These requirements now frequently comprise a third to two-thirds of total clinical trial cost. $^{15}$

Another example is the IRB system. IRBs are an important innovation that helps protect subjects and ensure adherence with national and international standards for biomedical ethics. ${ }^{16}$ Over the years, however, the number of IRBs has greatly proliferated and their role

${ }^{10}$ Kenneth Getz et al., Assessing the Impact of Protocol Design Changes on Clinical Trial Performance 15 AMERICAN JOURNAL OF THERAPEUTICS 450 (2008).

11 See Leila Duley et al., Specific Barriers to the Conduct of Randomized Trials, CliniCAL Trials 40, 44 (2008) (arguing that clinical trial regulations have made even "low cost" trials expensive).

12 See GAO, supra note 7, at 31 (citing industry analyst reports and a European Commission study that determined that the U.S. FDA began to demand more complex regulatory requirements in response to series of high-profile drug withdrawals between 1997 and 2001).

13 See Robert M. Califf, Clinical Trials Bureaucracy: Unintended Consequences of Well-Intentioned Policy, 3 CLINICAL TRIALS 496 (2006); Seth Glickman et al., Ethical and Scientific Implications of the Globalization of Clinical Research, 360 NEW ENGLAND JOURNAL OF MEDiCINE 816, 818 (2009).

${ }^{14} \mathrm{GAO}$, supra note 7. While the International Conference on Harmonization of Technical Requirements for Registration of Pharmaceuticals for Human Use's good clinical practice guidelines (ICH-GCP) are virtually silent on randomization, which is foundational to the scientific exercise of clinical trial design, their most extensive prescriptions are on data monitoring. See Guideline E6 of ICH-GCP SS 4.7, 5.18.

${ }^{15}$ Eisenstein, supra note 8.

${ }^{16}$ FDA investigational new drug regulations define institutional review boards as the oversight bodies "designated by an institution to review, approve initiation of and conduct periodic review of biomedical research involving human subjects. [Their p] rimary purpose is to assure protection of rights and welfare of human subjects." 21 CFR Part 56 (2010). 
has substantially expanded. IRBs once simply reviewed whether clinical testing met ethical standards; today, IRBs examine trial protocols to ensure written consent forms are sufficiently simple and clear, monitor the progress of testing, and maintain substantial records of activities. IRBs must meet in person and devote substantial time to their responsibilities. Each IRB imposes its own ethical standards, consistent with its mandate to protect local community standards. Regional and national review IRB processes are typically additional, rather than substitutes for local institutional review. ${ }^{17}$ With trials now often involving multiple, sometimes dozens and hundreds of sites, the costs and time imposed by the IRB system can be substantial.

Third, clinical studies are increasingly being conducted on a multi-country and multi-regional basis. This is done to support regulatory approval decisions in target markets and to tap larger pools of treatment naïve potential subjects. Regulatory barriers make conducting those global studies with a single clinical trial protocol difficult. ${ }^{18}$ The International Conference on Harmonization of Technical Requirements for Registration of Pharmaceuticals for Human Use (ICH) developed good clinical practice guidelines (ICHGCP) for the design, conduct, recording, and reporting of trials. These guidelines are now the global standard of how trials are run and a legal requirement in many countries. ${ }^{19} \mathrm{ICH}-$ GCP, however, leaves significant space for interpretation. Accordingly, many countries' regulations are based on ICH-GCP, but retain significant differences in their requirements.

Fourth, and finally, commercial practices, adopted to improve the speed and regulatory compliance of new product development trials, have transformed clinical trial practices generally, increasing their cost and complexity. A successful trial completed rapidly in patients with a common condition can lead to revenues of tens or hundreds of millions of dollars a year for a pharmaceutical company. Shorter clinical development preserves more of a marketed product's patent life. There are significant competitive advantages to being the earliest product entrant in a therapeutic class. Under this commercial model, speed and reductions in the risk of regulatory noncompliance are a greater priority than cost. Put in another way, the commercial aversion to the risks of avoidable clinical trial delays and regulatory non-compliance is significant given the size of the potential financial rewards that may be lost.

To ensure regulatory compliance, clinical trial protocol designs have become more ambitious and demanding. Consequently, clinical trials have more subjects and more sites per trial than previously. Between 1999 and 2005, the mean number of procedures performed on each study volunteer has increased almost nine percent annually across all phases and therapeutic areas. $^{20}$ The standard operating procedure for many commercial trials involve detailed data

\footnotetext{
${ }^{17}$ Duley, supra note 11 at 44.

18 See Mark Paxton and Jean-Louis Saillot, Industry Efforts on Simultaneous Global Development, 43 DRUG INFORMATION JOURNAL 339 (2009).

${ }^{19} \mathrm{ICH}-\mathrm{GCP}$ is somewhat controversial as some have argued that the guidelines are unscientific and place unnecessary resource pressures on noncommercial trials and developing country national regulatory authorities (NRAs), which were not involved in developing the guidelines. See Alex D. McMahon et al., The Unintended Consequences of Clinical Trials Regulations, 6 PLOS MEDICINE 1 (2009); David A. Grimes, The Good Clinical Practice guideline: A Bronze Standard for clinical research, 366 LANCET 172 (2005).

${ }^{20}$ Getz, supra note 10.
} 
collection, extensive auditing of all data points (key or otherwise), and close scrutiny that ensure the proffered evidence is confirmed, without necessarily achieving a corresponding improvement in patient safety or results. ${ }^{21}$ Many argue that commercial trials often "overinterpret" regulatory guidance and requirements, doing more than required and focusing on regulatory compliance instead of the scientific demands of the trial. ${ }^{22}$ The cost implications of such practices are significant.

Over the last twenty years, these commercial and regulatory pressures have led to a clinical trial support industry and a proliferation of new business models. Clinical trials are increasingly intermediated by commercial contract research organizations (CROs) that recruit clinicians and patients, and manage the day-to-day operations of clinical trials. ${ }^{23}$ CROs specialize in navigating the maze of clinical trial regulatory requirements and structures and producing trials that meet the needs of national regulatory authorities. Site management organizations (SMOs) coordinate with CROs to ensure rapid IRB approval and faster site initiation and patient recruitment. Data management organizations collect, monitor, and maintain clinical trial data and study records. These companies are increasingly part of the standard overhead for conducting clinical trials; it has become difficult to run global trials without their assistance.

These developments affect developed and developing countries alike. Developed country regulatory models and commercial clinical practices are often imported into developing countries and adopted for clinical trials for drugs and vaccines for neglected diseases. Developing country governments adopt the regulations and guidance of the FDA and European Medicines Agency (EMA) because they are publicly available and familiar to the commercial clinical trial sponsors that developing country governments hope to attract. Likewise, the same commercial clinical trial practices are employed broadly, including in highly cost sensitive clinical trials in neglected disease-endemic countries, because they are familiar, accepted practice. ${ }^{24}$

\section{Challenges of Clinical Development of Health Products for Neglected Diseases}

The challenges of clinical development are compounded in the context of neglected diseases by three additional factors: (A) the absence of research infrastructure and regulatory capacity in many neglected disease-endemic settings; (B) the particularly difficult regulatory

\footnotetext{
${ }^{21}$ For example, the standard operating procedures for many industry-sponsored trials call for every case report form to be reviewed in person by a monitor who physically visits the enrolling site - a very expensive practice. Califf, supra note 13 at 500. See also Salim Yusuf, Randomized Clinical Trials, Slow Death by a Thousand Unnecessary Policies, 171 CANAdiAn MediCAL Association JOURnAL 889, 890 (2004).

22 See, e.g., Salim Yusuf et al., Sensible Guidelines for the conduct of large randomized trials, 5 CLINICAL TRIALS 38-39 (2008); Eisenstein, supra note 8; Duley supra note 11.

${ }^{23}$ In 1991, academic health centers share of the clinical trials funding pie dropped from $80 \%$ in 1991 to $30 \%$ in 2002; 70 percent of drug companies employed CROs in their projects. CROs now represent a multi-billion dollar industry. Christopher-Paul Milne, Harbingers, or Harvesters of change? OUTSOURCING 13 (2004).

24 See, e.g., Mary Moran et Al., Wellcome Trust, The New Landscape of Neglected Disease Drug DEVELOPMENT 25-26 (2005)(noting that, in 2005, one third of PDPs used CROs to support their R\&D process, which generally charged full commercial rates for their services).
} 
and ethical challenges posed by neglected disease product development trials; and $(\mathrm{C})$ the frequent need to conduct these trials in multiple sites and jurisdictions. This section reviews each of these factors in depth.

A. Lack of regulatory capacity in neglected disease-endemic settings hinders trials and places subjects at risk

Clinical trials must be conducted where the burden of the relevant disease exists. Neglected diseases are endemic primarily in Africa, Asia, and tropical regions of the Americas, with a lower prevalence in the Middle East. ${ }^{25}$ Accordingly, approximately two thirds of the clinical trials for neglected diseases that initiated subject recruitment between 2003 and 2009 occurred in disease-endemic regions, with nearly a third of the total occurring in Africa. ${ }^{26}$

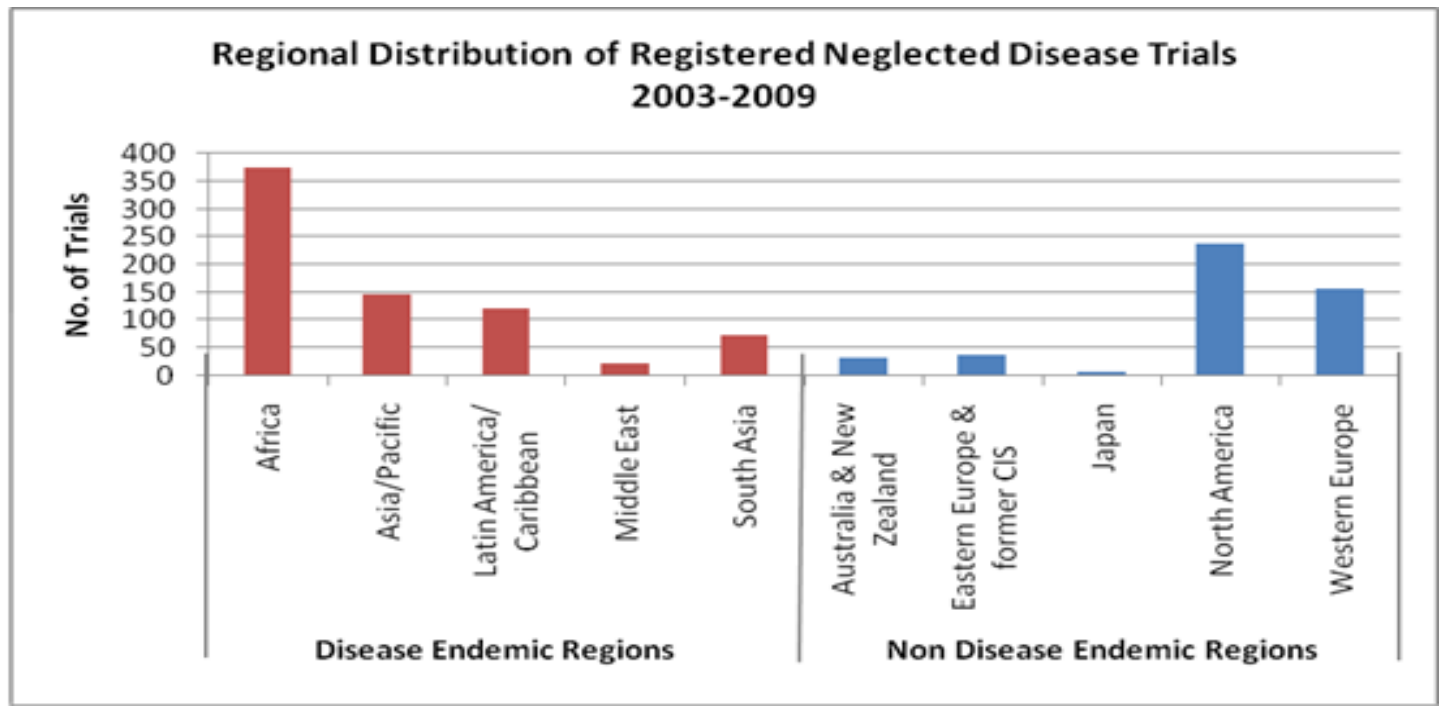

The geographic regions that have the highest neglected disease burden are also those with the most poorly resourced and inexperienced regulators and ethics committees. Many neglected disease-endemic countries, particularly in Africa, have weak or no national regulatory authorities (NRAs) and little ethical review capacity. ${ }^{27}$ Where NRAs do exist,

25 Philip Musgrove \& Peter Hotez, Turning Neglected Tropical Diseases into Forgotten Maladies, 28 LANCET 1691, 1693 (2009).

${ }^{26}$ Using data from Clinicaltrials.gov, our collaborators, Drs. Ernst Berndt, Iain Cockburn, and Fabio Thiers at the National Bureau of Economic Research, quantified country-specific participation in clinical trials initiating subject recruitment between January 1, 2003 and December 31, 2009 and aggregated them into geographic regions. Bioinformatics and keyword methods were employed to classify trials by type of intervention, sponsor, study phase, and therapeutic area. Information on trial size and number of sites is used to allocate subjects to countries. Proportion of trials of relevant studies not registered on Clinicaltrials.gov is not known, but is thought to be smaller post-2005, after the International Committee of Medical Journal Editors initiated a policy requiring investigators to deposit information about trial design into an accepted clinical trials registry before beginning patient enrollment.

${ }^{27}$ Diadié Maïga et al., Regulatory oversight of clinical trials in Africa: Progress over the past 5 years, VACCINE (2009) (citing a WHO Regional Office for Africa (WHO/AFRO) study that determined 36 percent of its member states lack IRBs); World Health Organization, Report on Workshop on Regulatory Procedures for Clinical Evaluation of Vaccine, Addis Ababa, 21-23 September, 2005 (concluding that only four of thirteen attending 
they often lack sufficient legal authority to approve clinical trial protocols, authorize importation of study products, inspect sites, or terminate trials. ${ }^{28}$ Even where the legal framework for clinical trial regulation exists, limited resources and training undermines the effectiveness of NRAs and IRBs. ${ }^{29}$ Regulators and ethics committees often lack sufficient personnel to review clinical trial protocols and inspect sites by accepted international standards and/or in a timely fashion.

Regulatory pathways and procedures in disease-endemic countries are frequently unclear and may change in unpredictable ways. National regulatory authorities and ethics committees have little interaction and duplicate each other's efforts; it may be difficult to determine their respective roles and responsibilities. ${ }^{30}$ Regulatory and ethics committees have highly variable practices, particularly with respect to trial monitoring. ${ }^{31}$ Ethics requirements are opaque and overlapping. Information on clinical trial regulatory requirements is not easily accessible to the public. Regulators' guidance may be unavailable or, when given, a moving target.

It is difficult to conduct ethical, sufficiently regulated trials in such environments. The lack of regulatory and ethics capacity undermines the safety of subjects and the validity and integrity of clinical data. ${ }^{32}$ The inability to understand local laws hinders trial planning, delays trial initiation and patient recruitment, and may lead to regulatory non-compliance. This situation presents challenges for sponsors committed to conducting ethical and sufficiently regulated clinical trials for neglected diseases. ${ }^{33}$ The risk of regulatory noncompliance and harm to subjects exposes trial sponsors and investigators to legal liability and reputational risk, deterring private investment.

The proportion of neglected diseases trials occurring in disease-endemic countries, nonetheless, increases for larger, late stage clinical trials. This reflects the reality that the definitive studies of the safety and efficacy of therapeutics must generally be conducted in the populations and environments for which they are intended. Late stage trials tend to involve a greater number of subjects and, often, more complex trial design and procedures. Accordingly, these trials place greater demands on local research and regulatory capacity.

governments had national regulatory authorities involved in clinical trials review, authorization of importation of clinical batches, and/or inspection of clinical trial sites); NETWORKING FOR ETHICS ON BIOMEDICAL RESEARCH IN AFRICA, FINAL REPORT 94 (2006) (determining that that 10 of 15 African countries assessed either lacked legal or regulatory requirements for the ethical conduct of human clinical research or had not implemented the legislation that existed).

${ }^{28}$ WORLD HEALTH ORGANIZATION, ASSESSMENT OF MEDiCinES REGULATORY SYSTEMS IN 22 WHO MEMBER STATES: A Summary OF Results (2009) (assessing 22 developing country NRAs in Asia, Africa, and Latin America between 2001 and 2005 and concluding that two-thirds of these countries had weak or no mechanisms for regulating clinical trials or exerting proper oversight on clinical investigation).

${ }^{29}$ Glickman, supra note 13 at 820 (monitoring guidelines are only effective to the degree to which they are implemented).

30 Maïga, supra note 27.

31 NETWORKING FOR ETHICS, supra note 27 at 95-97.

3221 CFR 312.120 (2009) (requiring, for admissibility, that foreign data come from clinical trials in which subjects gave their informed consent, an IRB approved and monitored the trial, and internationally recognized GCP were followed).

${ }^{33}$ Helen A. Fletcher et al., A New V accine for Tuberculosis: The Challenges of Development and Deployment, 6 BIOETHICAL INQUIRY 219, 224 (2009). 


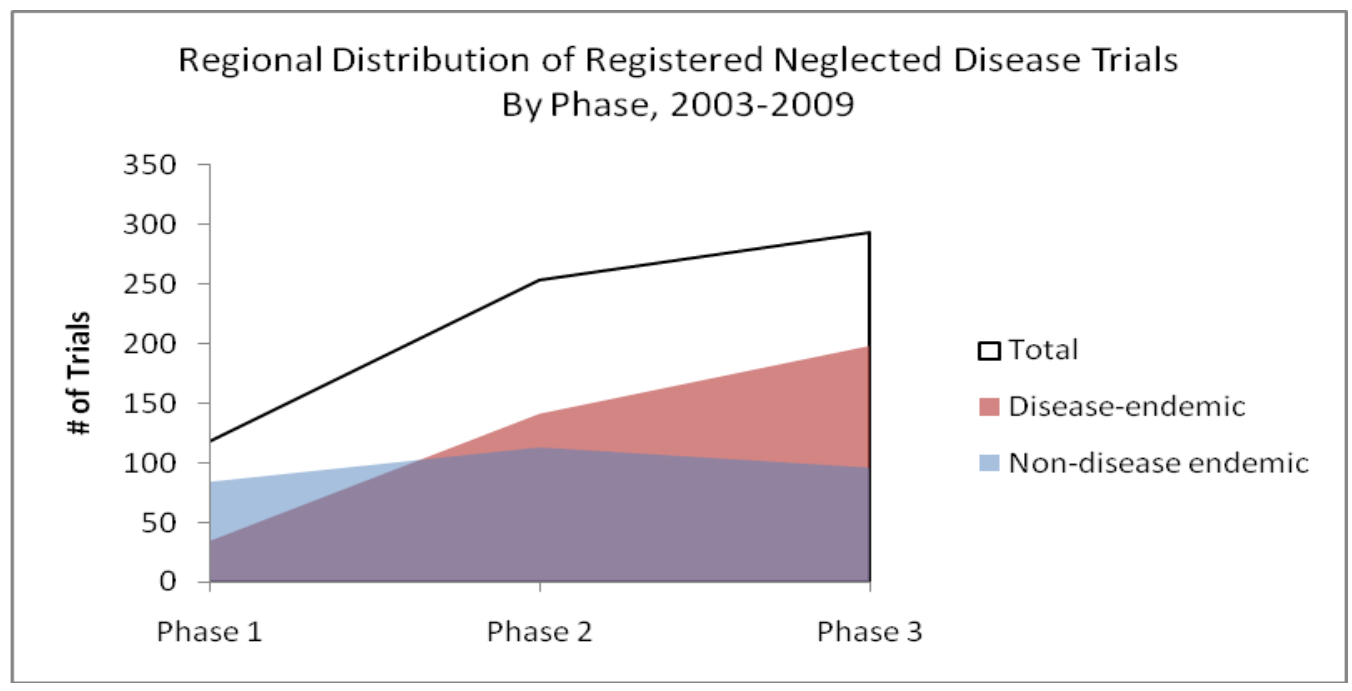

To minimize regulatory risk and uncertainty, many trial sponsors report seeking parallel trial registration with either the EMA or FDA, and to conduct the trial in a more developed neglected disease-endemic country such as South Africa or India. These high disease burden countries have the expertise and legal framework to conduct a more competent regulatory and ethics review, but, because of resource constraints, that review can take a disproportionately long time. Regulatory approval for trials in the U.S. and European Union (EU) can generally be obtained within 30-60 days. In many neglected disease-endemic countries, approval can take as long as 6-24 months. ${ }^{34}$ For products that require multiple trials to establish the safety and efficacy of the product in different subject populations, subsequent clinical trial application approvals may take as long as or longer than the original. ${ }^{35}$ Trial protocol amendment approvals that require a few weeks in developed countries can require as long as four months in these settings. ${ }^{36}$ In many cases, steps in the regulatory and ethical approval process need to be done in sequence, rather than simultaneously and in parallel, so that more than a year can pass between finalizing a trial protocol and the completion of all governmental and institutional regulatory and ethics

\footnotetext{
${ }^{34}$ Deborah Cook, Randomized Trials in Vulnerable Populations, 5 CLINICAL TRIALS 61 (2008) (noting that it took 918 months in some developing country trials to obtain import licenses as well as national regulatory approval). See, e.g., Kathryn Senior, Experts Warn of Regulatory Hurdles Stalling Drug Trials 8 THE LANCET INFECTION 281 (2008) (reporting that University of London Phase II and phase III rifamycin trials in South Africa were delayed two years due to regulatory hurdles and that a large scale Radboud University medical centre trial of high-dose rifampicin in Tanzania was delayed for a full year because of complications in the regulatory approval process).

35 One PDP has shared with CGD the regulatory review times for that PDP's various vaccine trials in South Africa, India, Kenya, Sweden, and Finland. Original CTA review times were 13.7 months in India, 10.2 months in Kenya, and between 5.8-7.3 months in South Africa versus 2.8 and 2.9 months in Sweden and Finland respectively. Approval times in South Africa for additional trials involving a product for which the RSA Medicines Control Council had previously approved a CTA ranged from 5.1 to 9.9 months. See PDP Regulatory Review time table (on file with author). ${ }^{36} I d$.
} 
processes. $^{37}$ Those delays not only prolong clinical trial initiation and patient recruitment, but also extend the time before new effective products may be registered.

B. Neglected disease trials in disease-endemic countries pose particularly difficult regulatory and ethical challenges

Clinical trials for health products for neglected diseases impose particularly difficult regulatory and ethical challenges that compound the problem of inexperienced and underresourced NRAs and IRBs. Those challenges are at least threefold.

First, the science is often difficult, imposing additional challenges for the inexperienced regulators and IRBs who must assess the scientific validity and the risk/benefit ratio of the proposed trials. ${ }^{38}$ Much remains unknown about many neglected diseases. For many of these diseases, there may be no validated surrogate marker or immunological correlate of protection. $^{39}$ Trials may require clinical endpoints that require significant time to develop, or are severe disease outcomes or mortality. The most likely scenario for many neglected disease trials is a partial success at best.

Some neglected diseases require multidrug regimens to address bacterial subpopulations or prevent the development of resistance. ${ }^{40}$ Testing drug candidates individually can add years to the development of effective combinations. ${ }^{41}$ Testing novel drugs together can make it difficult to assign side effects to a particular candidate drug or interaction between drugs. Development of regulatory guidelines for clinical trials of novel combination drug regimes remains a work in progress in the United States and will be limited to "dire" illnesses such as cancer, HIV/AIDS, and tuberculosis due to the inherent uncertainty and risks around testing drugs in combination. ${ }^{42}$ The conduct and regulation of novel combination product trials in the neglected disease-endemic environments will be a significant challenge.

Second, neglected disease trials present extreme versions of the already difficult ethical challenges of conducting clinical research in developing countries. ${ }^{43}$ Clinical trials often must be conducted with highly vulnerable clinical trial subjects in devastatingly poor settings in disease-endemic countries with little healthcare infrastructure. Wide disparities in the

${ }^{37}$ Duley, supra note 11 at 44; see also NETWORKING FOR ETHICS ON BIOMEDICAL RESEARCH IN AFRICA, FINAL REPORT 95-97 (2006) (outlining the sequential regulatory and ethics review processes, for example, in Nigeria, Tanzania, and Uganda).

38 See Ezekiel Emanuel et al., What makes clinical research in Developing Countries Ethical? 189 JOURNAL OF Infectious Disease 930 (2004); World Medical Association, DeClaration of Helsinki, Art 12, 21.

39 See, e.g., Fletcher et al., supra note 33 at 221.

40 See, e.g., Zhenkun Ma et al., Global Tuberculosis Drug Development Pipeline: the need and the reality, THE LANCET SERIES, at 5-6, available at http://www.thelancet.com/series/tuberculosis (last visited on May 19, 2010).

${ }^{41}$ Critical Path to TB Drug Regimens, Global Partners Join Forces to Speed Development of New TB Combinations, Press Release (Mar. 18, 2010) (announcing a partnership of the Bill \& Melinda Gates Foundation, the Global Alliance for TB Drug Development, the Critical Path Institute, and numerous pharmaceutical companies to pursue clinical development of TB drug candidates in combination, arguing that testing these products individually would add as much as 20 years to development time).

42 See Mark Schoofs, FD A is Easing Way for Drug Cocktails, THE WALl STREET JournAL, at A8 (Mar. 18, 2010).

43 See Glickman, supra note at 13; Emanuel et al., supra note 38. 
education, economic and social standing of investigators and subjects and the poor quality of local health care systems may jeopardize the rights of research participants. Subjects may not always understand the investigational nature of therapeutic products and the use of a placebo. If there is an existing drug or vaccine for the disease available, as is the case with tuberculosis, the ethics and science of the trial design are greatly complicated. ${ }^{44}$ The question of what qualifies as fully informed consent is not always simple.

The burden of neglected diseases falls disproportionately on infants and children; the subjects for clinical trials for neglected diseases are frequently pediatric.

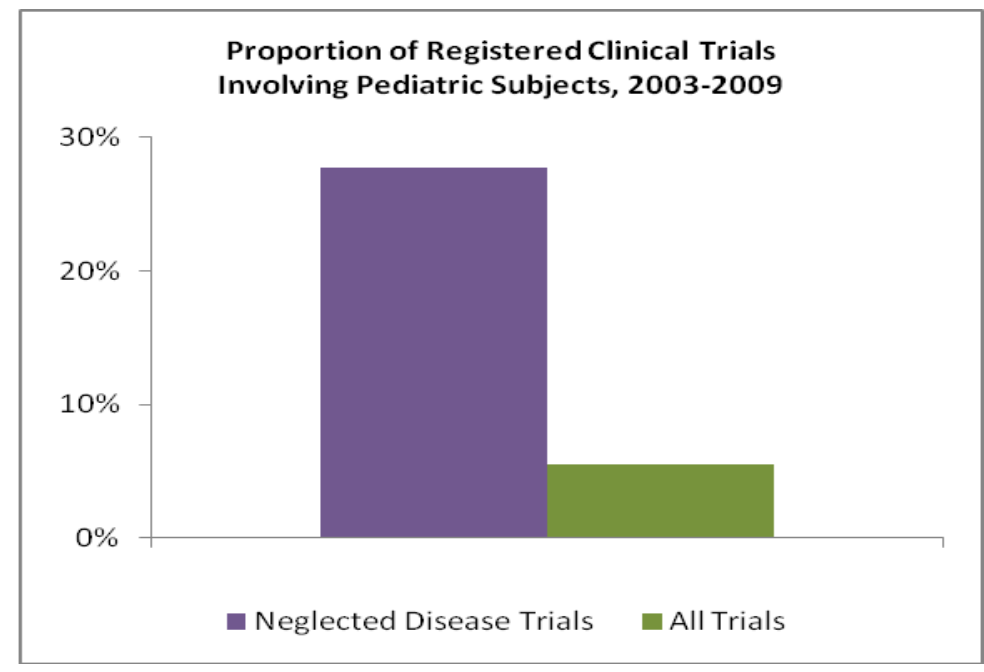

Pediatric trial subjects are particularly vulnerable and, thus, pose difficult ethical and operational challenges. Again, the majority of these difficult pediatric neglected disease clinical trials are occurring in Africa - the region with the least regulatory capacity and expertise to oversee them. ${ }^{45}$

\footnotetext{
${ }^{44}$ For diseases like HIV/AIDS and malaria, where no current vaccine exists, the ethics trial design focuses on appropriate sample size and placebo selection. Fletcher et al., supra note 33 at 221. In the case of tuberculosis, the inquiry is more complicated. There is an existing licensed TB vaccine - Bacille Calmette Guèrin (BCG). It is a widely used, live attenuated vaccine, but estimates of its efficacy range from 0-80 percent. Dixie E. Snider Jr., Ethical Issues in Tuberculosis Vaccine Trials, 30 CLINICAL INFECTIOUS DISEASES S271-275 (2000). The vaccine has efficacy against childhood TB, but this decreases over time and revaccination does not confer protection. The development of a new and more reliably efficacious and safer vaccine is necessary to control the global spread of tuberculosis. There have been questions whether new TB vaccines can be safely and effectively used in populations already immunized with the BCG vaccine, see Michael J. Brennan, The Tuberculosis Vaccine Challenge, 85 TUBERCULOSIS (EDINB) 7-12 (2005), and the ethics of trials designed to show the non-inferiority of new vaccines over BCG. Fletcher et al., supra note 33 at 222-23.

45155 of the 233 pediatric clinical trials for neglected diseases in disease-endemic countries registered on Clinicaltrials.gov and initiating recruitment between 2003 and 2009 occurred in Africa.
} 


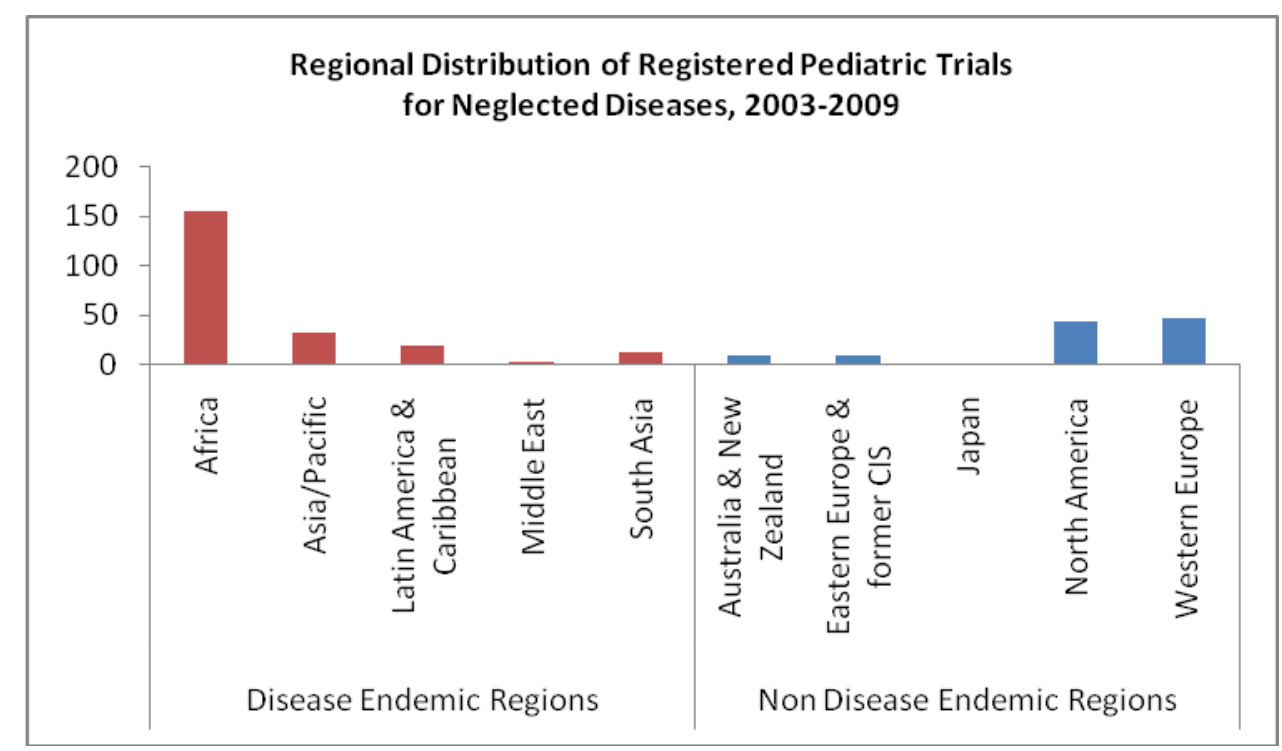

Third, most of the products in development to treat neglected diseases -76 percent - are vaccines. ${ }^{46}$ Vaccines trials impose additional regulatory and ethical challenges to clinical development. These trials must usually be conducted with healthy people, often children. The products are frequently scientifically complex, requiring determinations of host/pathogen interaction and immune response. Vaccines also introduce particular logistical challenges (for example, the need for refrigeration) and require infrastructure for their storage and administration. To demonstrate a sufficient immune response, the trials must frequently be large - involving tens of thousands of clinical trial subjects. ${ }^{47}$ The personal benefit to the clinical trial subjects may be limited and provisional, with the greater benefit of the product accruing to the community. PDPs report much longer delays in regulatory and ethics approvals of trials for vaccines than drug products in disease-endemic countries.

C. Many clinical trials for products for neglected diseases are multi-center and often multi-country.

The regulatory problems presented by the clinical development of neglected disease products in disease-endemic countries are compounded by the need to conduct many of these trials at multiple sites in multiple jurisdictions.

Close to half of the biopharmaceutical and vaccine trials for neglected diseases registered between 2003 and 2009 involved two or more trial sites and nearly one-third had sites in

\footnotetext{
46 Tufts Center for the Study of Drug Development, supra note 2.

${ }^{47}$ Accordingly, the clinical development of vaccines is also more expensive than drugs. For instance, a novel TB drug is estimated to cost at $\$ 115$ million to $\$ 240$ million, including cost of failure, but vaccine development from research and discovery through to product registration is estimated at $\$ 200$ to $\$ 500$ million, including cost of failure. See MAry Moran ET Al., The GeOrge Institute for International HeAlth, G-Finder REPORT: NegleCted Disease RESEARCH AND DEVElopMENT - HOW MuCH ARE We REALly SPENDING 45 (2008).
} 
multiple countries. ${ }^{48}$ The majority of multi-country product development trials for neglected diseases had all their sites within a single geographic region. ${ }^{49}$

These findings about clinical trials for neglected diseases differ from the general trend in global clinical trial activity in two respects.

First, a much smaller proportion of clinical trials overall had sites in more than one country (14 percent). This result suggests that clinical trial sponsors avoid conducting clinical trials in multiple countries, perhaps due to the need to coordinate multiple government and regulatory authorities and to pay additional fees and import duties. In contrast, the greater use of sites in more than one country in neglected disease trials most likely reflects either the limited site capacity in disease-endemic countries (requiring sites in other countries) and/or the need to test the candidate product in settings where different epidemiological, service delivery and socioeconomic conditions or strains of the disease exist.

Second, the overwhelming majority (83 percent) of multi-country clinical trials overall involved sites in multiple geographic regions. ${ }^{50}$ This suggests that, as a general matter, once a sponsor decides to run a trial with sites in more than one country, that sponsor chooses sites in countries with the most research capacity, favorable regulatory system, and/or patient recruitment potential. In contrast, the regional concentration of the sites in multi-country product development neglected disease clinical trials suggests that the choice of those sites is driven by presence of the disease burden rather than the regulatory or research qualities of the host country.

Whatever the motivation, the need to navigate regulatory and ethics requirements in multiple jurisdictions adds delays, costs, and uncertainty to the already time-consuming, costly, and risky clinical development process. ${ }^{51}$ Multiple regulators and IRBs reviewing the same protocols and consent forms wastes scarce in-country regulatory capacity and resources. ${ }^{52}$ Regulatory authorities and IRBs in different disease-endemic countries often impose different or inconsistent requirements or review timelines. ${ }^{53}$ Those inconsistent requirements

48 An analysis of Clinicaltrials.gov data reveals that 272 of the 902 biopharmaceutical and vaccine trials for neglected diseases between 2003 and 2009 were multi-country trials.

49 Our analysis of clinicaltrials.gov data indicates that 112 neglected disease trials were multi-region as well as multi-country. Our analysis of these multi-region trials has not broken down these trials by product type. Accordingly, all we can determine is that at least 155 of the 272 of multi-country neglected disease product development trials occur within a single region. The proportion may be higher.

${ }^{50}$ Our analysis of Clinicaltrials.gov data indicates that 8026 out of the universe of 66,169 registered trials initiating subject recruitment between 2003 and 2009 were trials involving sites in two or more countries. Of these 7110 multi-country trials, 5888 had sites in two or more geographic regions (Asia, Africa, Western Europe, etc.).

51 See Duley, supra note 11 (noting that large trials involving multiple developing countries, the collective process of obtaining regulatory and IRB approvals, importing drugs, and negotiating contracts can add 12-24 months to trials and millions of dollars in expenses).

52 Califf, supra note 13.

53 See WHO, TRREE-FOr AFrica AND the Institute of HEALTH LAW (UNIVERSity OF NeUCHÂTEL), THE ETHICAL AND REGULATORY REVIEW MECHANISMS OF CliniCAL TRIALS IN AFRICA: OVERVIEW OF THE SitUATION IN THE AVAREF MEMBERS COUNTRIES (2009) (revealing spectacular variety in the ethical and regulatory review mechanisms in assessed African countries); PAN AMERICAN HEALTH ORGANIZATION, 
necessitate multiple trial protocol submissions, resulting in divergent regulatory decisions and requests, which delay trial initiation. ${ }^{54}$ These regulatory differences frequently extend to adverse event reporting and other compliance requirements, increasing trial costs. The resulting regulatory cacophony affords no obvious benefit, in most cases, to scientific rigor, quality, or protection of trial subjects.

\section{A Sustainable Strategy for Bringing Clinical Trial Costs, Risks, and Finances into Better Balance}

Two substantial bottlenecks threaten clinical development of neglected disease products. First, the research and regulatory capacity in many neglected disease-endemic settings is not adequate to support the clinical trials that need to occur there in order to complete the development of these products. Second, even with expected attrition in the pipeline, current levels of financing are insufficient to support the clinical development of these products under current cost assumptions.

Part of the answer must be more funding for trials and the training programs, infrastructure, and sites in neglected disease-endemic settings necessary to run them pursuant to good clinical and laboratory practice standards. World Health Organization (WHO) technical assistance programs, such as the Special Program on Tropical Disease Research, and regional and disease-specific research and clinical trial capacity building efforts, such as the European and Developing Countries Clinical Trials Partnership and Malaria Clinical Trials Alliance, deserve further support.

Addressing these twin challenges of high costs and limited regulatory and research capacity, however, also requires new approaches. Global health budgets are tightening and new donor funding is scarce. Donor and sponsor insistence on approximating rich-country clinical development models under difficult poor-country conditions will only lead to a consequent escalation of delays, complications, and costs. A country-by-country approach to research and regulatory capacity building is not feasible.

The development of clinical trial practices and the creation of a regulatory environment that is more friendly to the conduct of clinical trials would result in multiple winners: trial subjects and patients, global health interests, the multinational pharmaceutical industry, and developing country governments interested in building regulatory capacity and infrastructure. More efficient clinical trial practices and defined and streamlined regulatory

REPORT ON THE ASSESSMENT OF THE SITUATION OF GOOD CLINICAL PRACTICES IN 12 LATIN AMERICAN COUNTRIES (2000) (finding that 10 of 12 Latin American countries with regulations in place on clinical trials had different content, requirements, and application regarding ethics committees, informed consent, research on vulnerable populations, and certification of researchers and research centers).

54 See Chris Granger, Duke Clinical Research Institute, Minimizing delays within regulatory agencies, presentation at Sensible Guidelines Conference (September 5, 2009), available at http://www.ctsu.ox.ac.uk/projects/sg (noting regional differences in regulatory delays in 5 trials with delays ranging from 15 to 90 days in the U.S., Japan, UK, Germany, and France for unapproved drugs as opposed to 39 days to 10 months for India, Argentina, and China). 
practices would improve the commercial viability of neglected disease product development projects and encourage private investment in clinical research capacity in disease-endemic countries.

This section describes two potential complementary approaches - sensible guidelines for neglected disease clinical trials and regional regulatory streamlining - for reducing unnecessary costs, risk, and delays in clinical trials for health products for neglected diseases without sacrificing scientific rigor, quality assurance, and the protection of trial subjects.

A. Sensible Practices for Clinical Trials for Products for Neglected Diseases in DiseaseEndemic Countries

Clinical trial sponsors and investigators may be in the best position, in the near term, to reduce clinical trials costs and delays for products for neglected diseases.

Several promising initiatives are assessing large, multi-center clinical trials to evaluate how they may be modified to reduce their cost without sacrificing scientific rigor, quality assurance, or the protection of trial subjects and patients.

- In 2007, clinical trial research groups from McMaster, Duke and Oxford Universities initiated the Sensible Guidelines for the Conduct of Clinical Trials Project. ${ }^{55}$ The Project advocates strategies for the simple trial design of large trials - widely applicable and as close to normal clinical practice as possible - in order to reduce costs and improve patient accrual. ${ }^{56}$ The project has released a series of papers and hosted conferences devoted to developing, inter alia, rational and risk-based approaches to documenting and evaluating safety and data monitoring. ${ }^{57}$

- Also in 2007, the FDA's Office of Critical Path Programs and Duke University launched a public-private partnership, the Clinical Trials Transformation Initiative (CTTI), to research and develop practices that, through broad adoption, would increase the efficiency and quality of clinical trials. ${ }^{58}$ The initial efforts of CTTI will focus on two projects: (1) improving the system for reporting serious adverse events to clinical investigators and (2) ways of improving clinical trial monitoring to eliminate those practices that do not ensure the reliability of data and trial participant protection.

\footnotetext{
55 http://www.ctsu.ox.ac.uk/projects/sg.

${ }^{56}$ Duley, supra note 11 at 40-41.

57 See, e.g., Yusuf et al., supra note 22; Cook, supra note 34; Colin Baigent et al., Ensuring trial validity by data quality assurance and diversification of monitoring methods, 5 CLINICAL TRIALS 49 (2008); Christopher Granger et al., Do we need to adjudicate major clinical events, 5 CLINICAL TRIALS 56 (2008); Eisenstein, supra note 8; Duley, supra note 11; Jane Armitage et al., The impact of privacy and confidentiality laws on the conduct of clinical trials, 5 CLINICAL TRIALS 70 (2008).

${ }^{58}$ Currently, over 50 organizations comprise CTTI, including U.S. government and international agencies, industry representatives (pharmaceutical, biotech, device, and clinical research organizations), patient and consumer representatives, professional societies, investigator groups, academic institutions, and other interested parties. See Clinical Trials Transformation Initiative, https:/ /www.trialstransformation.org/.
} 
This research is important and useful, but focuses on the demands of cardiovascular, cancer, and other industrialized world product clinical trials. It may not be necessarily applicable to clinical trials for vaccines or drugs for neglected diseases, given highly vulnerable and pediatric subjects, and trial sites, health care systems, and regulatory environments with limited capacity.

For instance, several proposals advocate the use of selective site visits combined with centralized monitoring and statistical sampling techniques to reduce expensive on-site auditing and data monitoring. ${ }^{59}$ The strategy is potentially consistent with ICH-GCP. ${ }^{60}$ It could allow more targeted use of monitoring resources to provide training at sites, improve adherence to protocol and procedures, and verify adequacy of site resources. ${ }^{61}$ Reducing the heavy monitoring burdens at sites might also allow sponsors to move from a payment per subject model of clinical trial practice to a more centralized model in which trial sites are paid only for the costs of the hosting center. One study modeled a large scale pharmaceutical industry phase III trial and determined that this strategy would result in a 21 percent cost reduction, which would increase to a 35 percent cost reduction when combined with moving to electronic data capture rather than paper case report forms. ${ }^{62}$ This is an exciting result, but more analysis and research is required to determine the applicability of strategies like centralized monitoring, statistical sampling, and electronic data capture to resource-poor neglected disease-endemic settings with minimal local regulatory oversight.

CGD is working to develop sensible guidelines for clinical trials for neglected disease products. We are bringing together respected experts from academic centers, local NRAs and IRBs, industry, and PDPs to adapt emerging research on efficient late stage clinical trial practices to the needs of the neglected disease product pipeline and the challenges of the regulatory pathways for these products. The resulting research and guidelines will benefit sponsors and investigators of clinical trials for these products by reducing unnecessary costs and delays and help inform capacity building efforts by WHO, funders, and developing country governments.

B. Regional regulatory pathways for the clinical development of health products for neglected diseases

A single, regional pathway with integrated regulatory and ethics reviews for clinical trials would have significant potential benefits for participating neglected disease-endemic countries.

\footnotetext{
59 See, e.g., Eisenstein, supra note 8; Baigent et al., supra note 57 at 49, 50; Yusuf, supra note 21 at 890; Califf, supra note 13 at 500 .

${ }^{60} \mathrm{ICH}-\mathrm{GCP}$ guidelines much favor on-site monitoring, but permit centralized monitoring. A combination of the two monitoring practices is entirely consistent with ICH-GCP requirements. See Section 5.18.3 of Guideline E6 of ICH-GCP.

${ }^{61}$ Baigent et al., supra note 57.

${ }^{62}$ Eisenstein, supra note 8.
} 
First, it would improve the quality of clinical trial regulation and the protection of the clinical trial subjects in participating disease-endemic countries by pooling their scarce regulatory resources and increasing regulator-to-regulator and regulator-to-IRB interaction. Such a regional pathway would also provide a platform for foundations, NGOs, governments, and intergovernmental entities seeking to support clinical trial regulatory capacity building in disease-endemic regions and developing countries, but without the resources to duplicate those investments on a country-by-country basis. ${ }^{63}$

Second, a regional, integrated pathway for regulation of clinical trials would help reduce inconsistency in regulatory and ethics requirements and their interpretation, as well as limit the number of regulatory and ethics review and compliance obligations. In doing so, such a pathway would expedite trial initiation and reduce the cost and uncertainty of conducting clinical trials in participating neglected disease-endemic countries.

Third, reductions in the cost, duration, and risks of conducting clinical trials in diseaseendemic countries would improve the use of scarce existing resources for neglected disease product clinical development, reduce barriers to private sector investment, and expedite patients' access to treatments. ${ }^{64}$ Given the scale of funding required for clinical development, modest improvements in efficiency could yield substantial savings that may be used to develop other products for neglected diseases. ${ }^{65}$

Finally, better protection for subjects and a more cost- and time-efficient regional regulatory approach with more certain review timelines and procedures would help attract private clinical trial activity to neglected disease-endemic regions and investment in local and regional research capacity. ${ }^{66}$ There is substantial and growing commercial interest in conducting biopharmaceutical clinical trials in developing countries to reduce the spiraling

\footnotetext{
${ }^{63}$ See OfFice of Inspector General, U.S. Department of Health AND Human Services, CHALlENGES TO FDA's ABILITY TO MONITOR AND INSPECT FOREIGN CliniCAL TRIALS, OEI-01-08-00510 (2010) (reporting FDA's interest in building the capacity of foreign regulators to help address the exponential growth in overseas clinical trial activity).

64 See Sarah Frew et al., A Business Plan to Help Fight Against Neglected Diseases, 28 LANCET 1760, 1763 (2009) (noting that many emerging economy companies regard neglected diseases as business opportunities); MORAN, supra note 23 at 65-66 (noting that existing neglect disease markets, particularly for TB, malaria, and possibly leishmaniasis, offer unexploited opportunities with comparable returns to average orphan markets that attract small firms, but that the barriers of conducting large-scale clinical development, inter alia, deter such investment).

${ }^{65}$ Dalberg Advisors recently estimated that \$6-10 billion is needed to complete the clinical development of the drugs in the pipeline for neglected diseases, even with expected rates of attrition. See Paul L. Herrling, Making Drugs Accessible to Poor Populations: A Funding Model, Global Forum UpDate On ReSEARCH FOr HeALTH 152 (2008) (citing a study, commissioned by International Federation of Pharmaceutical Manufacturers \& Associations (IFPMA) and Novartis).

${ }^{66}$ Ernst R. Berndt, The Globalization of Clinical Trials for New Medicines into Emerging Economies: Where are They Going and Why? (2007) (manuscript on file with author) (noting that speed of patient recruitment and completion of the trials is widely reported as a critical consideration in choosing a trial site). See THE GEORGE INSTITUTE, REgISTERING NEW DRUGS: THE AFriCAN CONTEXT (2010) (proposing regional centers of excellence to support clinical research for drugs to address diseases of the developing world).
} 
costs of clinical trials and improve the speed of patient recruitment. ${ }^{67}$ Clinical trial activity is an important and growing source of foreign direct investment for many developing countries. ${ }^{68}$ There are potential spillover benefits as well: diffusion of medical knowledge and effective medical practice; increased resources and training for hospitals, medical schools, and regional research centers; long-term development of domestic life sciences industries; and greater patient access to high-quality medical care. ${ }^{69}$ Accordingly, a regional regulatory pathway would help promote development of local innovative industry capacity.

Regional regulatory cooperation is not without its challenges. Countries value their sovereignty in regulatory affairs and the independence and local accountability of their regulatory authorities. Regional regulatory cooperation requires a supporting infrastructure - agreements and coordination mechanisms - to direct activities and support the exchange of confidential information, applications, and inspection reports. Generating multi-state regulatory architecture is already challenging; it may be doubly difficult for countries that may not have such regulatory infrastructure and legal frameworks domestically.

That said, there are several useful and successful precedents for such regional regulatory pathways involving both developed and developing countries.

\section{The EMA Centralized Procedure}

The EMA centralized procedure provides a single application, single evaluation, and a single review process allowing direct access to all national markets of the EU. ${ }^{70}$ It is an intriguing model for several reasons.

First, the principle motivation for establishing the centralized procedure was not regulatory harmonization, but rather the pooling of regional regulatory expertise on a difficult regulatory problem. European Community (EC) NRAs lacked expertise in the novel techniques needed to assess biotechnology products. ${ }^{71}$ The centralized procedure enabled these regulators to work together on biotechnology product registration applications with the intention of achieving a common decision. ${ }^{72}$ These circumstances bear similarities with the situation in many neglected disease-endemic countries around the difficult issues presented by many neglected disease product development trials.

\footnotetext{
${ }^{67}$ Fabio A. Thiers et al., Trends in the Globalization of Clinical Trials, NATURE REVIEW DRUG DisCOVERY (2007) (concluding that 24 of the fastest growing 25 countries in terms of clinical trial participation in 2007 were emerging markets, with China, Argentina, Russia, and India exhibiting high growth rates).

68 See, e.g., INDIAN MINISTRY OF COMMERCE \& INDUSTRY, REPORT OF THE TASK FORCE: STRATEGY FOR INCREASING EXPORTS OF PHARMACEUTICAL PRODUCTS 69 (2008) (estimating that the clinical trial activity in India will be worth $\$ 300$ million in 2010).

${ }^{69}$ Thiers et al., supra note 67; Glickman et al., supra note 13 at 818.

${ }^{70}$ Council Regulation (EEC) no. 2309/93 22 July 1993.

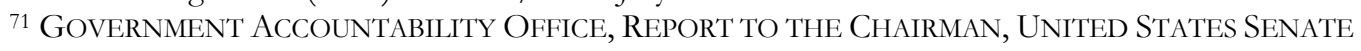
Committee on Labor and Human Resources, European Union Drug ApProval: OverviEW OF NEW European Medicines Evaluation AgenCy And Approval Process 4 (1996).

72 It was initially known as the "concertation procedure." EC Directive 87/22 for high-technology or biologically-derived products.
} 
Second, the centralized procedure did not require the harmonization or dissolution of participating national regulatory authorities, which is often a sensitive issue of national sovereignty and employment. ${ }^{73}$ However, for many of these products, the member states agreed on identical product information documents, which later became obligatory.

Third, the centralized procedure evolved relatively quickly. International and regional regulatory harmonization efforts are notoriously complex, expensive, and arduous. ${ }^{74}$ The EC had been working on pharmaceutical registration harmonization since 1975, but its efforts were focused on its slow developing mutual recognition process. ${ }^{75}$ The EC created the forerunner to the centralized procedure to address the biotechnology problem in 1987 and formalized it six years later. It was the first EU drug regulatory procedure in which no member state had issued an approval of a product before the procedure started. ${ }^{76}$

Fourth, the centralized procedure has been scalable. It was initially mandatory for a small, defined list of biotechnology and high-technology products and optional for all nonbiotechnological drugs considered potentially innovative. Over the years, this mandatory list has been expanded to include medicines for HIV/AIDS, cancer, diabetes, neurodegenerative diseases, all designated orphan medicines, and all veterinary medicines intended for use as performance enhancers.

Finally, the centralized procedure has been enormously successful. Within its first year of formal operation, two-thirds of the centralized applications that industry filed were done so voluntarily. ${ }^{77}$ The procedure effectively integrated the drug approval process for newer therapies that might have otherwise proved controversial across EU markets. ${ }^{78}$ The U.S. Government Accountability Office estimates that the centralized procedure saved an estimated 40 percent of the cost and, more importantly, greatly reduced approval times over obtaining separate marketing authorizations in, at that time, 15 EU member states. ${ }^{79}$

\footnotetext{
73 Alar Irs et al., Development of marketing authorization procedures for pharmaceuticals, in EVALUATING Pharmaceutical for Health Policy and Reimbursement (ed. Nick Freemantle and Susan Hill, 2004) (noting that in its initial form, normal national regulatory reviews occurred after the committee review). ${ }^{74}$ SUZANNE HILl \& KENT JOHNSON, UK DEPARTMENT FOR INTERNATIONAL DEVELOPMENT, EMERGING CHALLENGES AND OPPORTUNITIES IN DRUG REGISTRATION AND REGULATION IN DEVELOPING COUNTRIES (2004).

75 The mutual recognition process did not initially require participating states to accept as authoritative the product review conducted by other member state agencies and, consequently, each country continued to perform their own product review before coming to a decision regarding product approval. This duplication of effort among the different national regulatory authorities undermined the success of the process. Elaine M. Healy \& Kenneth Kaitin, The European Agency for the Evaluation of Medicinal Products' Centralized Procedure for Product Approval: Current Status, 33 DRUG INFORMATION JOURNAL 969, 970 (1999).

${ }^{76}$ Irs et al., supra note 73.

77 GOVERNMENT ACCOUNTABILITY OFFICE, supra note 71 at 12

${ }^{78} \mathrm{Id}$. at $1-4$.

${ }^{79} I d$. at 10.
} 
AVAREF

The WHO has supported the creation of the African Vaccine Regulatory Forum (AVAREF), a network of 19 countries that the WHO identified as likely settings for clinical trials of priority vaccines. One of the activities of AVAREF is an ad hoc joint regulatory and ethics review process for vaccine clinical trials in Africa. ${ }^{80}$ Working with trial sponsors, the WHO facilitated joint reviews by African NRAs and ethics committees of trial protocols in conjugate meningitis A and malaria vaccine clinical trials and joint inspections of the sites involved. The purpose of the AVAREF initiative was to address the lack of technical expertise and capacity in the underlying countries. The results of its joint review have not been binding. Nonetheless, the process has been widely viewed as successful, improving the capacity and coordination of participating NRAs and ethics committees and encouraging the use of defined review timelines and common documentation. ${ }^{81}$

The WHO has similar plans to conduct joint evaluation of clinical trial applications in Asia. The procedure would follow the format used in AVAREF, including both IRBs and NRAs, a single format for the applications, and a joint report that participating countries would use as the basis for their independent regulatory decision.

\section{The Integrated Research Application System in the United Kingdom}

The Integrated Research Application System (IRAS), launched in 2008 in the United Kingdom, also provides a promising model of a centralized, integrated clinical trial regulatory and ethics review pathway. IRAS provides a single, integrated application point for regulatory and ethics review of multi-site and single site clinical trials in the UK; only issues of local ethical concern are assessed by local IRBs. The system reduces bureaucratic burden, particularly for multi-site studies. It helps eliminate duplication; study wide checks are performed only once. The system also improves national ethics review consistency and creates a single, secure online database and document repository.

Borrowing heavily from these examples, an illustration of a potential model for a regional, integrated regulatory pathway is represented below.

\footnotetext{
80 See Diadié Maïga et al., Joint Reviews and inspections: Strategic forms of Collaboration for Strengthening the oversight of vacine clinical trials in Africa, Vaccine (2009).

81 See id.
} 
A Proposal for a Regional Integrated Regulatory Pathway for Clinical Trials

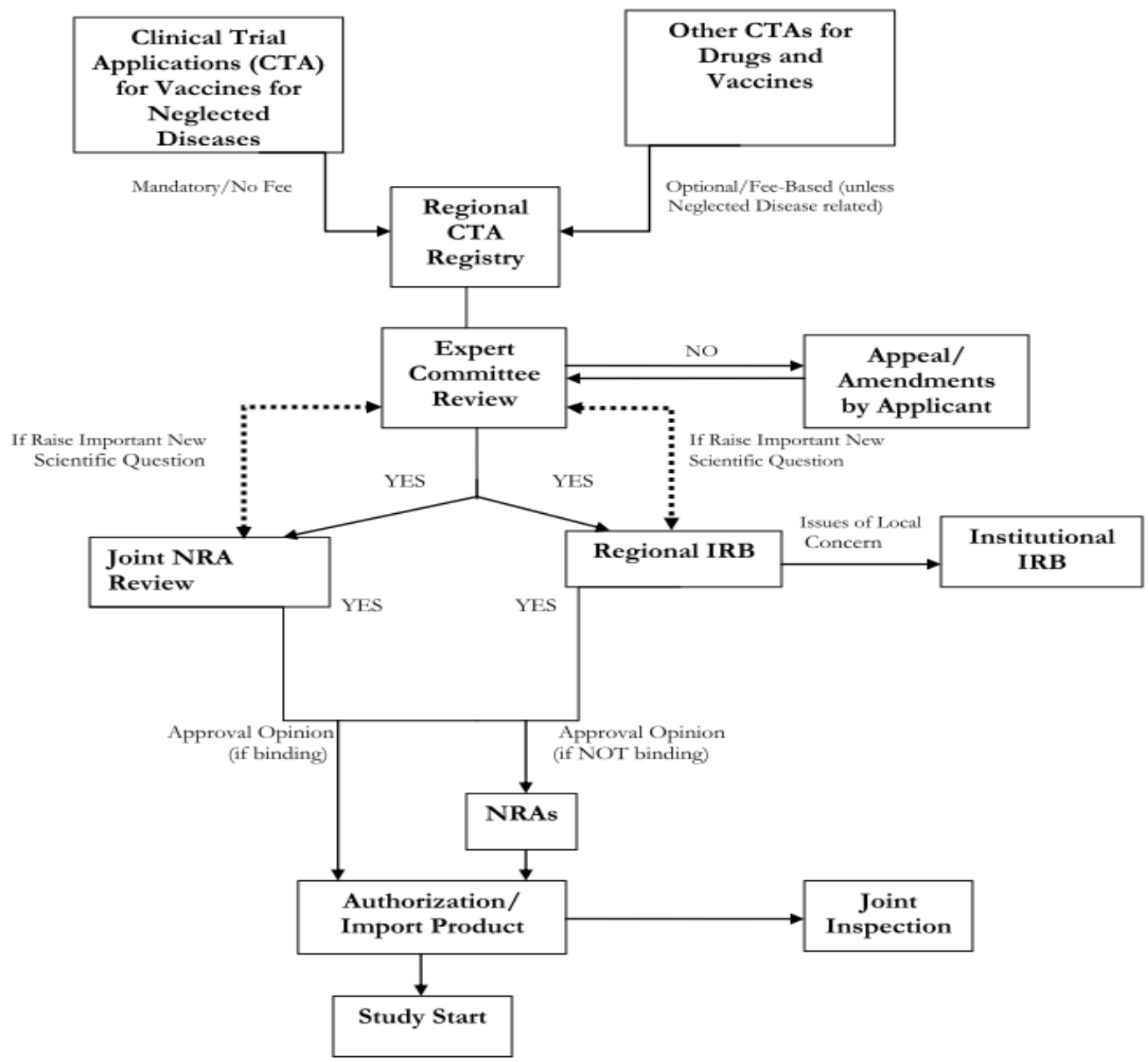

The pathway depicted in this figure is a centralized procedure/joint review model in which participating NRAs and IRBs would jointly review clinical trial applications and perform inspections of trial sites.

The benefits of this approach are threefold. First, it does not require eliminating the underlying NRAs or harmonizing national regulatory requirements. Second, a centralized procedure/joint review model promotes the buy-in, capacity building, and regional integration of participating countries by requiring their collective effort to review applications and perform site inspections. Third, the integrated nature of the review promotes collaboration between NRAs and IRBs and avoids duplication in their efforts.

In the model depicted above, the review of clinical trial applications would be performed by an expert committee comprised of representatives of the participating country NRAs and IRBs with assistance, as needed, from outside experts from the FDA, EMA, or other 
qualified regulatory authorities. ${ }^{82}$ The application would include the investigator's brochure, a signed protocol and amendments (if any), written information given to the trial subject (including the consent form), the safety dossier, sample case and trial monitoring reports, certifications and information concerning the investigational product, resumes and evidence regarding the qualification of the investigators, information on the financial aspects of the trial, and the relevant agreements between the involved parties (investigator, sponsor, and CROs (if involved)).

The expert committee would conduct a scientific review of the application and issue its opinion to the joint review committee and regional IRB. If the opinion is positive, the joint review committee members and IRBs would have a defined period of time to complete their review of the submission, conduct a site inspection, and authorize or deny the trial and importation and release of the study product. In this model, the regulatory and ethics determinations would occur in parallel and simultaneously. The ethics review would be conducted by regional IRB, leaving only issues of local relevance to national and local IRBs.

For qualifying applications, participating NRAs and IRBs would use a common set of regulatory and ethics requirements and protocol and safety monitoring report formats. Reviews would be completed pursuant to public, defined regulatory and ethics review timelines. ${ }^{83}$ The pooling of regional regulatory resources should allow the setting of more ambitious timeframes than would otherwise be possible on a national basis.

At least initially, the process would be mandatory for only one category of products: vaccines for neglected diseases. Our analysis of the trials registered on Clinicaltrials.gov suggests there is a strong regional orientation to product development trials for neglected disease. The bulk of the products in development for neglected diseases are vaccines. These products present particularly difficult regulatory and ethics challenges to NRAs and IRBs, which would benefit from the pooled regulatory resources of this regional pathway. Like the centralized procedure, this regulatory pathway could be expanded over time to include other products.

Neglected disease-endemic countries' participation in this regional regulatory review process should be voluntary and its results, at least initially, non-binding. As the benefits of the model are demonstrated to its participants, the process should become more formal and binding. Long-term, successful intergovernmental regulatory cooperation requires a binding legal framework. ${ }^{84}$

82 Julie Milstein \& Lahouari Belharbi, Regulatory Pathways for vaccines for developing countries, 82 BULLETIN OF WORLD HEALTH ORGANIZATION 128, 132 (2004)(arguing that a collective, expert committee approach would expand the ability of NRAs in this region to address the needs of the specific epidemiological situation of these countries). Ideally, the EMA and/or FDA would enter into a memorandum of understanding with the participating governments to provide that necessary technical and/or financial support at expert committee level.

83 Participating NRAs and manufacturers and trial sponsors seeking to use the regional regulatory pathway would need to enter into the necessary confidentiality agreements to allow sharing of proprietary information between national regulatory authorities. See Maïga et al., supra note 80.

${ }^{84}$ Irs et al., supra note 73 . A binding multilateral regulatory cooperation agreement could be used in this case to create the centralized procedure/joint review contemplated here. Under this model, the joint review process 
Research conducted in disease-endemic countries should contribute to research capacity development and the strengthening of the regulatory and ethics systems in the countries concerned. A streamlined, regional regulatory pathway with more certain regulatory timelines would hold material value for pharmaceutical firms and their investors, which could be harnessed to generate private-sector funding or cross-subsidies for that pathway or development of products for neglected diseases. One possible approach is that commercial clinical product development projects could be permitted to use the centralized regulatory pathway in exchange for a fee or other contribution. ${ }^{85}$ Use of that pathway for clinical development of global health products could be free or heavily discounted. Another possibility would be that CROs could register with the regulatory pathway to provide heavily discounted or free services to neglected disease product development projects in exchange for using the streamlined central regulatory pathway for their full-fare private clinical development projects. Fees and the increased commercial clinical trial activity that result from a more certain, expedited regional regulatory pathway would help induce country participation in the regional regulatory pathway.

The regional regulatory pathway could evolve from existing regional regulatory networks (e.g., AVAREF), link to regional, intergovernmental economic or public health organizations (e.g., the Southern African Development Community (SADC), Pan American Health Organization (PAHO), or the Association of Southeast Asian Nations (ASEAN)), or pooled procurement initiatives. The creation of a new free-standing institution to manage this regional regulatory pathway would not be necessary. It would also complement and build upon ICH and regional regulatory harmonization efforts and regional research and clinical trial capacity building programs.

Generating a design for this proposed pathway would require an evidence-based approach and the strong support of its prospective constituents. CGD is bringing together experts and stakeholders from national regulators, ethics committees, contract research organizations, industry, PDPs, the WHO, and academic centers to: determine their motivations and needs for regional regulatory streamlining and cooperation; develop legal and institutional frameworks for the regulatory pathway; assess possible links to existing regional regulatory networks or regional, intergovernmental economic or public health organizations; and determine the technical support and transition costs - in time and money - required to move to a regional regulatory pathway.

would function like an arbitration clause or optional protocol of an international treaty. Signatory states enter into the treaty voluntarily and, in doing so, commit to abide by the results of future dispute settlement arising from the treaty, pursuant to its terms. Here, the parties would enter into a binding multilateral regulatory cooperation agreement and agree to abide the results of the joint review process. Failure to abide with provision, after appeal procedure, could lead to the state's expulsion from participation in the regional regulatory pathway, rather than financial penalties.

85 The use of review fees, in exchange for more certain review timelines, was used to improve regulatory capacity in the United States. The Prescription Drug User Fee Act (PDUFA) was enacted in 1992 and renewed in 1997 (PDUFA II), 2002 (PDUFA III), and 2007 (PDUFA IV, 21 U.S.C. \$S 379g-h, authorizes FDA to collect fees from companies that produce certain human drug and biological products. FDA sets the fees on a yearly basis. 


\section{Moving Towards Solutions}

An estimated one billion people, including 400 million children, suffer from one or more of neglected diseases. Many of these diseases exact a large and lethal toll, with tuberculosis and malaria alone killing an estimated 2.6 million people annually. ${ }^{86}$ Other neglected diseases are less deadly, but disable, deform, and increase their sufferers' vulnerability to other infectious diseases like HIV/AIDS. These diseases adversely affect pregnancies and child development, undermine worker productivity, and perpetuate the cycle of poverty, insecurity, and infirmity in the communities in which they are endemic. ${ }^{87}$ In sum, neglected diseases rob the world's poorest communities of their hope for a better future.

There is hope, however. The pipeline of health products to treat, prevent, or diagnose neglected diseases is fuller than it has been for many years. The challenges of achieving that potential are manifold and significant. Late stage clinical development of these candidate products will be slow and expensive, perhaps prohibitively so. Trials must be conducted with highly vulnerable subjects in environments with limited research and regulatory capacity and, often, across multiple jurisdictions with conflicting rules, standards, and procedures.

Increased funding for late stage clinical trials is certainly needed, but there must also be greater attention paid to how these trials and their regulatory pathways can be improved to reduce unnecessary costs, delays, and risks to subjects.

This paper describes a two-pronged strategy that would help achieve these goals and have compound benefits. Regulatory capacity building and streamlining would encourage donor and private investment in addressing diseases of the poor. Regional, sustainable approaches to clinical trial research and regulation in disease-endemic countries would better engage and equip local regulators and ethics committees to support the development of health products for neglected diseases and protect subjects. More favorable regulatory environments in developing countries may encourage indigenous innovation in health technologies to meet local needs. Finally, a regional approach would help pool and improve developing countries' clinical trial regulation at a time when FDA, EMA, and other developed country regulators do not have the resources or legal authority to address the exponential growth in trials conducted overseas. ${ }^{88}$

86 WHO, Global tuberculosis control: A short update to the 2009 report (2009) and WHO, World malaria report 2009 (2009).

${ }^{87}$ Peter J. Hotez et al., The antipoverty vaccines. 24 VACCINE 5787 (2006).

88 See OFFICE OF THE INSPECTOR GENERAL, supra note 63 (reporting that 80 percent of FY 2010 FDA approved marketing applications for drugs and biologics contained data from foreign clinical trials, but that FDA only inspects 0.7 percent of foreign clinical trial sites involved). 